\title{
Time-dependent density-functional theory for strong-field multiphoton processes: Application to the study of the role of dynamical electron correlation in multiple high-order harmonic generation
}

\author{
Xiao-Min Tong ${ }^{*}$ and Shih-I Chu \\ Department of Chemistry, University of Kansas, and Kansas Center for Advanced Scientific Computing, Lawrence, Kansas 66045
}

(Received 16 April 1997; revised manuscript received 26 August 1997)

\begin{abstract}
We present a self-interaction-free time-dependent density-functional theory (TDDFT) for nonperturbative treatment of multiphoton processes of many-electron atomic systems in intense laser fields. The theory is based on the extension of the time-dependent Kohn-Sham formalism. The time-dependent exchange-correlation potential with proper short- and long-range behavior is constructed by means of the time-dependent optimized effective potential (TDOEP) method and the incorporation of an explicit self-interaction correction (SIC) term. The resulting TDOEP-SIC equations are structurally similar to the time-dependent Hartree-Fock equations, but include the many-body effects through an orbital-independent single-particle local time-dependent exchangecorrelation potential. We also introduce a generalized pseudospectral time-propagation method, allowing optimal spatial grid discretization, for accurate and efficient numerical solution of the TDOEP-SIC equations. The theory is applied to the study of the role of dynamical electron correlation on the multiple high-order harmonic generation $(\mathrm{HHG})$ processes of He atoms in intense laser fields. We also perform a detailed study of the mechanisms responsible for the production of the higher harmonics in He atoms observed in a recent experiment that cannot be explained by the single-active-electron model. We found that both the dynamical electron correlation and the $\mathrm{He}^{+}$ion are important to the generation of the observed higher harmonics. The present TDDFT is thus capable of providing a unified and self-consistent dynamical picture of the HHG processes. [S1050-2947(98)04601-0]

PACS number(s): 42.50.Hz, 32.80.Wr, 32.80.Qk, 71.15.Mb
\end{abstract}

\section{INTRODUCTION}

The study of the nonlinear response of atoms and molecules in intense laser fields is a subject of much current interest in atomic, molecular, and optical physics. To describe such strong-field multiphoton processes using an $a b$ initio wave-function approach, it is necessary to solve the time-dependent Schrödinger equation of many-electron systems, which is far beyond the capability of current computational technology. Even for the two-electron systems in strong fields, time-dependent $a b$ initio wave-function studies are only at the beginning stage [1] and much remains to be explored. For multielectron systems (mainly rare-gas atoms), a time-dependent method being successfully used is the socalled single-active-electron (SAE) approximation with a frozen core [2,3], involving the numerical solution of the single-electron time-dependent Schrödinger equation. The SAE method has been shown to be valuable in providing insights on atomic multiphoton dynamics such as multiple high-order harmonic generation (HHG) [2,3]. Within the SAE model, however, the electron correlation, the excitedstate resonances, and the core excitation cannot be explicitly treated. It is desirable to explore more complete formalisms for detailed treatment of strong-field processes, taking into account both the dynamical electron correlation and the structure of the excited states and at the same time allowing for the core excitation of many-electron systems.

\footnotetext{
*Permanent address: Institute of Physics, P.O. Box 603, Chinese Academy of Science, Beijing 100 080, People's Republic of China.
}

In this paper we address such a nonperturbative formalism based on the extension of the time-dependent densityfunctional theory (TDDFT). The TDDFT is a nontrivial extension of the steady-state DFT of Hohenberg, Kohn, and Sham $[4,5]$ to the time domain [6-9]. The central result of the TDDFT is a set of time-dependent Kohn-Sham equations that are structurally similar to the time-dependent HartreeFock equations but include in principle all the many-body effects through a local time-dependent exchange-correlation (XC) potential. With the exception of the recent pioneering work of Ullrich, Grossmann, and Gross [9], most applications of the TDDFT fall into the regime of linear or nonlinear response in weak fields for which the perturbation theory is applicable. Recently, Telnov and Chu [10] have presented an alternative nonperturbative formulation of TDDFT based on the extension of the generalized Floquet formalism [11], allowing exact transformation of the time-dependent KohnSham equations into an equivalent time-independent Floquet matrix eigenvalue problem. Such a TDDFT-Floquet formalism provides a time-independent approach for nonperturbative treatment of multiphoton processes of many-electron systems in the presence of intense periodic or multicolor laser fields. In this paper, we focus on the time-dependent approach for the numerical solution of time-dependent KohnSham-like equations in arbitrarily time-dependent fields.

In the context of DFT, it is well known [12] that the XC energy functionals commonly used, such as the local spindensity approximation (LSDA) [13] or the more refined generalized gradient-correction approximation (GGA) [14-16], suffer a severe deficiency, namely, the XC potential decays exponentially and does not possess the correct long-range $(-1 / r)$ behavior. As a result, while the total energies of the 
ground-state atoms predicted by the GGA energy functionals are rather accurate, the ionization potentials obtained from the highest occupied orbital energies are not satisfactory $[12,17]$. Such a problem can be attributed to the existence of self-interaction energy in the conventional XC energy functionals used in the DFT formalisms. For quantitative treatment of ionization and multiphoton ionization processes, it is necessary to extend the DFT to properly account for the long-range $\mathrm{XC}$ potential such that both the ionization potential and the excited-state properties can be described more accurately. In a recent paper we addressed such a problem and presented an extension of the steady-state DFT for accurate treatment of the ionization potentials and the photoabsorption spectrum of autoionizing resonances [18]. The results are in good agreement with available experimental data as well as configuration-interaction calculations [18]. The method is based on the extension of the recent Krieger-LiIafrate (KLI) [19] semianalytic treatment of the optimized effective potential formalism [20,21] along with the use of an explicit self-interaction-correction (SIC) term [22]. A similar KLI-SIC procedure has been recently proposed independently by Chen et al. [23], but it has been applied to the study of ground-state energies and ionization potentials only. In the present paper, we extend such a KLI-SIC technique to the time-dependent domain. We note that a related timedependent KLI theory has been recently proposed by Ullrich, Grossmann, and Gross [9]. In the latter work [9], the Hartree-Fock (nonlocal) exchange energy functional form (instead of the explicit SIC form in our work) is used in the construction of time-dependent optimized effective potential. As shown in the recent steady-state DFT works [18,23], the use of the SIC form in the KLI calculations involves only orbital-independent single-particle local potentials and is thus computationally more efficient and yet maintains high accuracy. The extension of such a KLI-SIC procedure to the time domain is described in Sec. II.

The motivation of this paper is thus threefold. First, we present a TDDFT with optimized effective potential and selfinteraction correction in Sec. II for the general nonperturbative treatment of the multiphoton dynamics of many-electron atomic systems in the presence of intense laser fields, taking into account proper long-range behavior of the XC potential. Second, we outline a generalized pseudospectral timedependent method recently introduced [24] for efficient and accurate treatment of the time-dependent KLI-SIC equations in Sec. III. Such a time propagation procedure has been shown to be capable of providing significant improvement of the quality of the wave functions over those obtained by the conventional equal-spacing spatial discretization techniques and thereby giving rise to more accurate HHG spectra calculated [24]. Finally, we apply the TDDFT in Sec. IV to the nonperturbative study of $\mathrm{HHG}$ of $\mathrm{He}$ atoms in intense laser fields. A comparison with experimental data is made and the role of the dynamical electron correlation in the production of higher harmonics is explored in depth.

\section{TIME-DEPENDENT DFT WITH OPTIMIZED EFFECTIVE POTENTIAL AND SELF-INTERACTION CORRECTION}

\section{A. Time-dependent density-functional theory}

We first outline below the basic equations of TDDFT $[6,8]$. The central theme of the TDDFT involves a set of time-dependent one-electron Schrödinger-like Kohn-Sham (KS) equations for $N$-electron atomic systems (in atomic units),

$$
\begin{aligned}
i \frac{\partial}{\partial t} \psi_{i \sigma}(\mathbf{r}, t)= & \hat{H}(\mathbf{r}, t) \psi_{i \sigma}(\mathbf{r}, t) \\
= & {\left[-\frac{1}{2} \nabla^{2}+v_{e f f, \sigma}(\mathbf{r}, t)\right] \psi_{i \sigma}(\mathbf{r}, t), } \\
& i=1,2, \ldots, N_{\sigma}
\end{aligned}
$$

where $v_{e f f, \sigma}(\mathbf{r}, t)$ is the time-dependent effective potential depending upon the total electron density and $\sigma$ is the spin index. The total number of electrons in the system is $N=\Sigma_{\sigma} N_{\sigma}$, where $N_{\sigma}\left(=N_{\uparrow}\right.$ or $\left.N_{\downarrow}\right)$ is the total number of electrons for a given spin $\sigma$. Within the single determinant approximation, the total $N$-electron wave function can be expressed as

$$
\Psi(t)=\frac{1}{\sqrt{N !}} \operatorname{det}\left[\psi_{1} \psi_{2} \cdots \psi_{N}\right]
$$

and the total electron density at time $t$ is determined by the single-electron orbital wave functions $\left\{\psi_{i \sigma}\right\}$ as

$$
\rho(\mathbf{r}, t)=\sum_{\sigma} \sum_{i=1}^{N_{\sigma}} \rho_{i \sigma}(\mathbf{r}, t)=\rho_{\uparrow}(\mathbf{r}, t)+\rho_{\downarrow}(\mathbf{r}, t),
$$

where $\rho_{i \sigma}(\mathbf{r}, t)=\psi_{i \sigma}^{*}(\mathbf{r}, t) \psi_{i \sigma}(\mathbf{r}, t)$. The effective potential $v_{e f f}(\mathbf{r}, t)$ in Eq. (1) can be written in the general form

$$
v_{e f f, \sigma}(\mathbf{r}, t)=v_{e x t}(\mathbf{r}, t)+\frac{\delta J[\rho]}{\delta \rho_{\sigma}(\mathbf{r}, t)}+v_{x c, \sigma}(\mathbf{r}, t),
$$

where $\quad J[\rho]=\frac{1}{2} \iint \rho(\mathbf{r}, t) \rho\left(\mathbf{r}^{\prime}, t\right) /\left|\mathbf{r}-\mathbf{r}^{\prime}\right| d \mathbf{r} d \mathbf{r}^{\prime} \quad$ is the electron-electron Coulomb interaction energy, $v_{\text {ext }}(\mathbf{r}, t)$ is the "external" potential due to the interaction of the electron with the external electromagnetic field and the nucleus, and $v_{x c, \sigma}(\mathbf{r}, t)$ is the time-dependent exchange-correlation potential.

The set of equations (1) is an initial-value problem. In performing these TDDFT calculations, the crucial element is the input of reliable time-dependent exchange-correlation potential and the development of an efficient and accurate numerical procedure for the time propagation of the set of equations (1). In Sec. II B below we discuss a timedependent optimized effective potential formalism with KLISIC for constructing $v_{e f f}(\mathbf{r}, t)$. The time-propagation method used in this work is described separately in Sec. III.

\section{B. Time-dependent optimized effective potential formalism with self-interaction correction}

As is well known [12], the effective potential $v_{\text {eff }}$ in Eq. (4) for the steady-state case, obtained by the functional derivative of the commonly used explicit energy functional $E_{x c}$ such as the LSDA or GGA, contains the spurious selfinteraction part and does not possess the correct long-range Coulomb tail behavior. This imposes severe limitations on 
the applicability of DFT to excited-state and ionization problems. In the time-independent DFT, several approaches have been suggested to remove the self-interaction problem [12]. Perdew and Zunger [22] proposed the SIC version of a given approximate exchange-correlation energy functional $E_{x c}\left[\rho_{\uparrow}, \rho_{\downarrow}\right]$ :

$$
E_{x c}^{S I C}\left[\rho_{\uparrow}, \rho_{\downarrow}\right]=E_{x c}\left[\rho_{\uparrow}, \rho_{\downarrow}\right]-\sum_{\sigma} \sum_{i=1}^{N_{\sigma}}\left\{J\left[\rho_{i \sigma}\right]+E_{x c}\left[\rho_{i \sigma}, 0\right]\right\},
$$

where $\rho_{i \sigma}$ is the single-electron density of the $i$ th KS spin orbital. In the limit that exact $E_{x c}\left[\rho_{\uparrow}, \rho_{\downarrow}\right]$ is used, the SIC term $\left\{J\left[\rho_{i \sigma}\right]+E_{x c}\left[\rho_{i \sigma}, 0\right]\right\}$ vanishes. Thus this SIC correction term can be also considered as a measure of the deviation of a given approximate $E_{x c}\left[\rho_{\uparrow}, \rho_{\downarrow}\right]$ from the exact result. The use of the SIC energy functional $E_{x c}^{S I C}\left[\rho_{\uparrow}, \rho_{\downarrow}\right]$ [Eq. (5)], however, leads to different potentials for different orbitals. Such an orbital dependence of the one-electron potentials causes the orbitals to be nonorthogonal. Additional effort must be taken to achieve orthogonal SIC spin orbitals.

Another promising approach for improving $E_{x c}\left[\rho_{\uparrow}, \rho_{\downarrow}\right]$ is provided by the so-called optimized effective potential (OEP) method $[20,21]$. In this approach, one solves a set of oneelectron equations, similar to the KS equations,

$$
\begin{gathered}
\hat{H}_{O E P} \phi_{i \sigma}(\mathbf{r})=\left[-\frac{1}{2} \nabla^{2}+V_{\sigma}^{O E P}(\mathbf{r})\right] \phi_{i \sigma}(\mathbf{r})=\varepsilon_{i \sigma} \phi_{i \sigma}(\mathbf{r}), \\
i=1,2, \ldots, N_{\sigma} .
\end{gathered}
$$

(To distinguish from the time-dependent wave functions $\left\{\psi_{i \sigma}\right\}$, the time-independent wave functions will be denoted by $\left\{\phi_{i \sigma}\right\}$.) The optimized effective potential $V_{\sigma}^{O E P}(\mathbf{r})$ is obtained by the requirement that the spin orbitals $\left\{\phi_{i \sigma}\right\}$ in Eq. (6) are those that minimize the total energy functional $E\left[\left\{\phi_{i \uparrow}, \phi_{j \downarrow}\right\}\right]$ :

$$
\frac{\delta E^{O E P}\left[\left\{\phi_{i \uparrow,} \phi_{j \downarrow}\right\}\right]}{\delta V_{\sigma}^{O E P}(\mathbf{r})}=0,
$$

with

$$
\begin{aligned}
E^{O E P}\left[\left\{\phi_{i \uparrow}, \phi_{j \downarrow}\right\}\right]= & T_{s}\left[\left\{\phi_{i \uparrow}, \phi_{j \downarrow}\right\}\right]+J\left[\left\{\phi_{i \uparrow}, \phi_{j \downarrow}\right\}\right] \\
& +E_{x c}\left[\left\{\phi_{i \uparrow}, \phi_{j \downarrow}\right\}\right]+\int v_{e x t}(\mathbf{r}) \rho(\mathbf{r}) d^{3} \mathbf{r},
\end{aligned}
$$

where $T_{s}$ is the kinetic-energy functional of the noninteracting electrons. Equation (8) for the energy functional $E^{O E P}\left[\left\{\phi_{i \uparrow}, \phi_{j \downarrow}\right\}\right]$ has the same form as that of the Hohenberg-Kohn energy functional since the total energy is a functional of the density $\rho$ and the density is determined by the spin orbitals $\left\{\phi_{i \uparrow}, \phi_{j \downarrow}\right\}$.

While the physical idea of the OEP method is simple and appealing, Eq. (7) leads to an integral equation that is computationally impractical to solve. Recently, Krieger, $\mathrm{Li}$, and Iafrate [19] have worked out an accurate semianalytic procedure to circumvent this difficulty, reducing the determination of $V_{\sigma}^{O E P}$ to the solution of simple linear equations. Ullrich, Grossmann, and Gross [9] have further extended the OEPKLI procedure to the time-dependent problems. In these steady-state [17,19] or time-dependent [9] KLI calculations, the Hartree-Fock (nonlocal) exchange energy functional is used in the construction of the optimized effective potentials. In a recent work [18], we showed that the KLI procedure can be further simplified considerably by the use of the SIC form, Eq. (5), without the need of using the nonlocal Hartree-Fock energy functional, in the construction of the optimized effective potential. Such an OEP-KLI-SIC procedure uses only orbital-independent single-particle local potential and is thus computationally more efficient and yet maintains high accuracy in the calculation of the groundstate energies, ionization potentials as well as the singly excited autoionizing resonances [18]. In the following, we extend the time-independent OEP-KLI-SIC procedure $[18,23]$ to the time domain. The advantage of using the explicit SIC form is particularly evident in the time-dependent problems since the effective single-particle potential is to be calculated for each small time step and an efficient procedure for generating the time-dependent OEP is very desirable.

First we consider the adiabatic approximation for the construction of the time-dependent XC potential $v_{x c, \sigma}(\mathbf{r}, t)$ in Eq. (4). Within such a framework, $v_{x c, \sigma}(\mathbf{r}, t)$ can be written as the functional derivative of the $x c$ energy $E_{x c}$ with respect to the density:

$$
v_{x c, \sigma}(\mathbf{r}, t)=\left.\frac{\delta E_{x c}\left[\rho_{\uparrow}, \rho_{\downarrow}\right]}{\delta \rho_{\sigma}}\right|_{\rho_{\sigma}=\rho_{\sigma}(\mathbf{r}, t)} .
$$

The simplest possible form is the adiabatic local spin-density approximation (ALSDA), in the $x$-only limit,

$$
v_{x, \sigma}(\mathbf{r}, t)=-\left[\frac{6}{\pi} \rho_{\sigma}(\mathbf{r}, t)\right]^{1 / 3}
$$

A more refined GGA energy functional such as that of Becke [14] and Lee, Yang, and Parr [15] can be used in Eq. (9). In the adiabatic approximation, the $\mathrm{XC}$ potential is local in time, i.e., no memory effect. Such an approximation is expected to be valid if the time dependence of $\rho_{\sigma}$ is sufficiently slow. In practice, it still gives quite good results even for the rapid time-dependent case. Similar to the steady-state case, however, the time-dependent XC potentials obtained by either ALSDA or the adiabatic GGA, etc., do not have the correct long-range Coulombic $(-1 / r)$ behavior. Thus the electrons are too weakly bound and the ionization potentials are significantly lower than those of the corresponding real atoms. In the following, we extend the time-independent OEP-KLI-SIC method [18] to the time-dependent problem, allowing the elimination of these problems.

The time-dependent Kohn-Sham-like equations with the OEP and SIC can be expressed as 


$$
\begin{aligned}
i \frac{\partial}{\partial t} \psi_{i \sigma}(\mathbf{r}, t)= & \hat{H}(\mathbf{r}, t) \psi_{i \sigma}(\mathbf{r}, t) \\
= & {\left[-\frac{1}{2} \nabla^{2}+V_{S I C, \sigma}^{O E P}(\mathbf{r}, t)\right] \psi_{i \sigma}(\mathbf{r}, t), } \\
& i=1,2, \ldots, N_{\sigma}
\end{aligned}
$$

where $V_{S I C, \sigma}^{O E P}(\mathbf{r}, t)$ is the time-dependent OEP with SIC, which can be written (similarly to the time-independent case) in the general form

$$
V_{S I C, \sigma}^{O E P}(\mathbf{r}, t)=v_{e x t}(\mathbf{r}, t)+\int \frac{\rho\left(\mathbf{r}^{\prime}, t\right)}{\left|\mathbf{r}-\mathbf{r}^{\prime}\right|} d^{3} \mathbf{r}^{\prime}+V_{x c, \sigma}^{S I C}(\mathbf{r}, t) .
$$

We now consider two different routes for the determination of $V_{S I C, \sigma}^{O E P}(\mathbf{r}, t)$; both lead to the same final expressions. The first route is to start from the adiabatic approximation and the time-dependent effective potential $V_{S I C, \sigma}^{O E P}$ in Eq. (11) is to be determined in such a way that the time-dependent orbital wave functions $\left\{\psi_{i \sigma}(\mathbf{r}, t)\right\}$ are those that minimize the total energy functional $E^{O E P}$ at any given time $t$ :

$$
\left.\frac{\delta E^{O E P}\left[\left\{\psi_{i \sigma}\right\}\right]}{\delta V_{S I C, \sigma}^{O E P}}\right|_{\psi_{i \sigma}=\psi_{i \sigma}(\mathbf{r}, t), \rho_{i \sigma}=\rho_{i \sigma}(\mathbf{r}, t)}=0
$$

Here $E^{O E P}$ is given by an expression similar to Eq. (8) except that the orbital wave functions and the density are now explicit functions of both $\mathbf{r}$ and $t$. The time-dependent OEP with SIC can now be written as

$$
\begin{aligned}
V_{x c, \sigma}^{S I C}(\mathbf{r}, t)= & \sum_{i} \frac{\rho_{i \sigma}(\mathbf{r}, t)}{\rho_{\sigma}(\mathbf{r}, t)}\left\{v_{i \sigma}(\mathbf{r}, t)+\left[\bar{V}_{x c, i \sigma}^{S I C}(t)-\bar{v}_{i \sigma}(t)\right]\right\} \\
v_{i \sigma}(\mathbf{r}, t)= & \frac{\delta E_{x c}^{S I C}\left[\rho_{\uparrow}, \rho_{\downarrow}\right]}{\delta \rho_{i \sigma}}=\frac{\delta E_{x c}\left[\rho_{\uparrow}, \rho_{\downarrow}\right]}{\delta \rho_{\sigma}}-\int \frac{\rho_{i \sigma}\left(\mathbf{r}^{\prime}, t\right)}{\left|\mathbf{r}-\mathbf{r}^{\prime}\right|} d^{3} \mathbf{r}^{\prime} \\
& -\frac{\delta E_{x c}\left[\rho_{i \sigma}, 0\right]}{\delta \rho_{i \sigma}(\mathbf{r}, t)},
\end{aligned}
$$

and

$$
\begin{gathered}
\bar{V}_{x c, i \sigma}^{S I C}(t)=\left\langle\psi_{i \sigma}\left|V_{x c, \sigma}^{S I C}(\mathbf{r}, t)\right| \psi_{i \sigma}\right\rangle, \\
\bar{v}_{i \sigma}(t)=\left\langle\psi_{i \sigma}\left|v_{i \sigma}(\mathbf{r}, t)\right| \psi_{i \sigma}\right\rangle .
\end{gathered}
$$

In deriving Eq. (15), use has been made of the explicit SIC expression in Eq. (5) with the density now depending on time.

An alternative route for deriving the time-dependent optimized effective potential with SIC is to implement the explicit SIC expression into the time-dependent OEP formalism recently developed by Ullrich, Grossmann, and Gross [9]. This formulation is more general and does not need to make the adiabatic approximation, and it also allows for the inclusion of the memory effect in principle. However, as will be shown below, when the commonly used explicit XC energy functionals such as those of the LDA, GGA, or Hartree-
Fock approximation are used, the final expressions reduce to those derived by the adiabatic approximation given above, namely, Eqs. (14)-(17).

In the time-dependent OEP formulation, the timedependent OEP with SIC is to be determined in such a way that the time-dependent orbital wave functions $\left\{\psi_{i \sigma}(\mathbf{r}, t)\right\}$ render the total actional functional $A\left[\left\{\psi_{i \sigma}(\mathbf{r}, t)\right\}\right]$ stationary, i.e.,

$$
\frac{\delta A\left[\left\{\psi_{i \sigma}(\mathbf{r}, t)\right\}\right]}{\delta V_{S I C, \sigma}^{O E P}(\mathbf{r}, t)}=0
$$

Following a procedure similar to the time-dependent OEPKLI scheme [9], one obtains the general expression for the time-dependent $\mathrm{XC}$ potential,

$$
\begin{aligned}
V_{x c, \sigma}(\mathbf{r}, t)= & \sum_{i} \frac{\rho_{i \sigma}(\mathbf{r}, t)}{\rho_{\sigma}(\mathbf{r}, t)} \frac{1}{2}\left\{v_{i \sigma}(\mathbf{r}, t)+v_{i \sigma}^{*}(\mathbf{r}, t)\right\} \\
& +\sum_{i} \frac{\rho_{i \sigma}(\mathbf{r}, t)}{\rho_{\sigma}(\mathbf{r}, t)}\left\{\bar{V}_{x c, i \sigma}-\frac{1}{2}\left[\bar{v}_{i \sigma}+\bar{v}_{i \sigma}^{*}\right]\right\} \\
& +\frac{i}{4 \rho_{\sigma}(\mathbf{r}, t)} \sum_{i} \nabla^{2} \rho_{i \sigma}(\mathbf{r}, t) \\
& \times \int_{-\infty}^{t} d t^{\prime}\left[\bar{v}_{i \sigma}\left(t^{\prime}\right)-\bar{v}_{i \sigma}^{*}\left(t^{\prime}\right)\right],
\end{aligned}
$$

where the last term contains the memory effect. Here

$$
v_{i \sigma}(\mathbf{r}, t)=\frac{\delta A_{x c}}{\psi_{i \sigma}^{*} \delta \psi_{i \sigma}}, \quad v_{i \sigma}^{*}(\mathbf{r}, t)=\frac{\delta A_{x c}}{\psi_{i \sigma} \delta \psi_{i \sigma}^{*}}
$$

and $A_{x c}$ is the $\mathrm{XC}$ action functional. In the present work, we shall use the following SIC expression for $A_{x c}$ :

$$
A_{x c}^{S I C}=\int_{-\infty}^{t^{\prime}} d t E_{x c}^{S I C}\left[\left\{\rho_{i \sigma}(\mathbf{r}, t)\right\}\right]
$$

from which we obtain

$$
v_{i \sigma}(\mathbf{r}, t)=v_{i \sigma}^{*}(\mathbf{r}, t)=\frac{\delta E_{x c}^{S I C}}{\delta \rho_{i \sigma}} .
$$

Note that $v_{i \sigma}$ is now a real function of $\mathbf{r}$ and $t$. Thus the memory term in Eq. (19) vanishes identically. The final expression for $V_{x c, \sigma}^{S I C}(\mathbf{r}, t)$ in Eq. (19) now reduces to the same form of Eq. (14). Thus both derivations lead to the same final results when the KLI procedure and explicit XC energy functional forms are used.

Note that in Eq. (14), the last two terms $\bar{V}_{S I C, \sigma}^{i}(t)$ and $\bar{v}_{i \sigma}(t)$ are constants, although the values of $\bar{V}_{S I C, \sigma}^{i}$ are unknown. The KLI method suggests a way to calculate $\bar{V}_{S I C, \sigma}^{i}(t)-\bar{v}_{i \sigma}(t)$ through a solution of the linear equations

$$
\sum_{i=1}^{N_{\sigma}}\left[\delta_{j i, \sigma}-M_{j i, \sigma}(t)\right]\left[\bar{V}_{S I C, \sigma}^{i}(t)-\bar{v}_{i \sigma}(t)\right]=\bar{V}_{j \sigma}^{s}(t)-\bar{v}_{j \sigma}(t),
$$

$$
j=1,2, \ldots, N_{\sigma}
$$


where

$$
M_{j i, \sigma}(t)=\int \frac{\rho_{j \sigma}(\mathbf{r}, t) \rho_{i \sigma}(\mathbf{r}, t)}{\rho_{\sigma}(\mathbf{r}, t)} d^{3} \mathbf{r}
$$

and

$$
\bar{V}_{i \sigma}^{s}(t)=\left\langle\psi_{i \sigma}\left|\sum_{j=1}^{N_{\sigma}} \frac{\rho_{j \sigma}(\mathbf{r}, t) \cdot v_{j \sigma}(\mathbf{r}, t)}{\rho_{\sigma}(\mathbf{r}, t)}\right| \psi_{i \sigma}\right\rangle
$$

We can now solve Eq. (11) and construct the optimized effective potential through Eqs. (12)-(17) and (23)-(25). We shall call this time-dependent procedure the TD-KLI-SIC method below.

\section{GENERALIZED PSEUDOSPECTRAL TIME-PROPAGATION METHOD FOR NUMERICAL SOLUTION OF TIME-DEPENDENT KOHN-SHAM-LIKE EQUATIONS}

In this section we discuss the numerical procedure for the solution of the set of time-dependent Kohn-Sham-like OEPKLI-SIC equations (11). The commonly used procedures for the time-dependent propagation of the Schrödinger equation employ equal-spacing spatial grid discretization [2,3,25-28]. For processes such as high-order harmonic generation, very accurate time-dependent wave functions are required to achieve convergence, since the intensity of various harmonic peaks can span a range of many orders of magnitude. Highprecision accuracy is generally more difficult to achieve by the equal-spacing spatial-grid-discretization time-dependent techniques. Due to the Coulomb singularity at the origin, the equal-spacing spatial-grid methods require a large number of grid points to achieve converged results. To achieve higher accuracy for the time-dependent wave-function propagation, we have recently introduced a numerical procedure [24] that consists of the following two basic elements. (i) A generalized pseudospectral technique $[29,30]$ is used for optimal grid discretization of the radial coordinates: denser mesh near the origin and short distances. The number of grid points required is generally considerably smaller than those used by the equal-spacing discretization methods. Yet higher accuracy in wave functions and therefore HHG spectra can be achieved, since the physically more important short-range regime is more accurately treated by this method [24]. The generalized pseudospectral method $[29,30]$ also has been recently applied successfully to the time-independent nonHermitian Floquet studies of atomic $[29,31]$ and molecular [32] processes in strong fields. It was found that the method is capable of providing high accuracy for both low-lying and highly excited complex quasienergy resonance states. (ii) A split-operator technique in the energy representation is introduced for efficient time propagation of the wave functions. In this work, we extend this procedure to the numerical solution of the time-dependent KLI-SIC equations. In the following we outline the basic procedure involved. A detailed description of the method can be found in Ref. [24].

Consider the solution of the time-dependent one-electron Kohn-Sham-like equation with OEP and SIC for $N$-electron systems in linearly polarized fields $(\mathbf{F} \| \mathbf{z})$,

$$
\begin{gathered}
i \frac{\partial}{\partial t} \psi_{i \sigma}(\mathbf{r}, t)=\hat{H} \psi_{i \sigma}(\mathbf{r}, t)=\left[\hat{H}_{0}+\hat{V}\right] \psi_{i \sigma}(\mathbf{r}, t), \\
i=1,2, \ldots, N_{\sigma} .
\end{gathered}
$$

Here $\hat{H}_{0}$ is the unperturbed Hamiltonian with an optimized effective potential and SIC at $t=0$, and $\hat{V}$ is the electronfield interaction and the residual time-dependent optimized effective potential with SIC:

$$
\begin{gathered}
\hat{H}_{0}(\mathbf{r})=-\frac{1}{2} \frac{d^{2}}{d r^{2}}+\frac{\widehat{L^{2}}}{2 r^{2}}+V_{S I C, \sigma}^{O E P}(\mathbf{r}, 0), \\
\hat{V}(\mathbf{r}, t)=-\mathbf{F} \cdot \mathbf{r} g(t)+V_{S I C, \sigma}^{O E P}(\mathbf{r}, t)-V_{S I C, \sigma}^{O E P}(\mathbf{r}, 0),
\end{gathered}
$$

where $F$ is the electric-field amplitude and $g(t)$ the total time-dependent part of the laser field. For the one-color field, $g(t)=f(t) \sin (\omega t)$, where $f(t)$ is the envelope function. We shall extend the second-order split-operator technique in spherical coordinates $[25,26]$ for the time propagation of the Kohn-Sham-like equations:

$$
\begin{aligned}
\psi_{i \sigma}(\mathbf{r}, t+\Delta t) \simeq & e^{-i \hat{H}_{o} \Delta t / 2} e^{-i \hat{V}(r, \theta, t+\Delta t / 2) \Delta t} e^{-i \hat{H}_{0} \Delta t / 2} \psi_{i \sigma}(\mathbf{r}, t) \\
& +O\left(\Delta t^{3}\right)
\end{aligned}
$$

Note that such an expression is different from the previous split-operator techniques [25,26,33], where $\hat{H}_{0}$ is usually chosen to be the radial kinetic-energy operator and $\hat{V}$ the remaining Hamiltonian depending on the spatial coordinates only. The use of the energy representation in Eq. (29) allows the explicit elimination of the undesirable fast-oscillating high-energy components and speeds up considerably the time propagation [24].

To pursue the time propagation, we expand the singleelectron wave function $\psi_{i \sigma}(\mathbf{r}, t)$ in Legendre polynomials [24-26],

$$
\psi_{i \sigma}\left(r_{i}, \theta_{j}, t\right)=\sum_{l=0}^{l_{\max }} g_{l}\left(r_{i}\right) P_{l}\left(\cos \theta_{j}\right)
$$

where the $P_{l}$ 's are the normalized Legendre polynomials. $g_{l}(r, t)$ can be determined accurately by the Gauss-Legendre quadrature

$$
g_{l}\left(r_{i}\right)=\sum_{k=1}^{L+1} w_{k} P_{l}\left(\cos \theta_{k}\right) \psi_{i \sigma}\left(r_{i}, \theta_{k}, t\right),
$$

where $\left\{\cos \theta_{k}\right\}$ are the $L+1$ zeros of the Legendre polynomial $P_{L+1}\left(\cos \theta_{k}\right)$ and $\left\{w_{k}\right\}$ are the corresponding quadrature weights. The propagation in $\hat{H}_{0}$ energy space can now be expressed as

$$
e^{-i \hat{H}_{0} \Delta t / 2} \psi_{i \sigma}\left(r_{i}, \theta_{j}, t\right)=\sum_{l=0}^{l_{\max }}\left[e^{-i H_{l}^{0} \Delta t / 2} g_{l}\left(r_{i}, t\right)\right] P_{l}\left(\cos \theta_{j}\right),
$$

with 


$$
H_{l}^{0}=-\frac{1}{2} \frac{d^{2}}{d r^{2}}+\frac{l(l+1)}{2 r^{2}}+V_{S I C, \sigma}^{O E P}(\mathbf{r}, 0)
$$

Note that in Eq. (32), each partial-wave component $g_{l}$ is propagated independently under individual $\hat{H}_{l}^{0}$ energy space, leading to efficient propagation of the total wave function.

The key step in the time propagation of Eq. (32) is to construct the evolution operator

$$
e^{-i H_{l}^{0} \Delta t / 2} \equiv S(l)
$$

through an accurate representation of $\hat{H}_{l}^{0}$. Here the generalized pseudospectral method $[24,29,30]$ is used for an optimal grid discretization and for accurate and efficient solution of the eigenproblem:

$$
\hat{H}_{l}^{0}(r) \chi_{l}(r)=\varepsilon \chi_{l}(r)
$$

The first step is to map the semi-infinite domain $[0, \infty]$ or $\left[0, r_{\text {max }}\right]$ onto the finite domain $[-1,1]$ using a nonlinear mapping $r=r(x)$, followed by the Legendre or Chebyshev pseudospectral discretization [29,30,34]. This allows for denser grids near the origin, leading to more accurate eigenvalues and eigenfunctions and the use of a considerably smaller number of grid points than those of the equal-spacing grid methods. A suitable algebraic mapping for atomic structure calculations is provided by the form $[29,30,34]$

$$
r=r(x)=L \frac{1+x}{1-x+\alpha},
$$

where $L$ and $\alpha=2 L / r_{\max }$ are mapping parameters. The introduction of the nonlinear mapping, however, usually leads to either an asymmetric or a generalized eigenvalue problem. Such undesirable features may be removed by the use of a symmetrization procedure recently introduced $[29,30]$. Thus, by defining

$$
\phi_{l}(r)=\sqrt{r^{\prime}(x)} \chi_{l}(r(x))
$$

one finds that the transformed Hamiltonian possesses the symmetrized form

$$
\hat{H}_{l}^{0}(x)=-\frac{1}{2} \frac{1}{r^{\prime}(x)} \frac{d^{2}}{d x^{2}} \frac{1}{r^{\prime}(x)}+V_{l}(r(x)),
$$

where $V_{l}=l(l+1) / 2 r^{2}+V_{S I C, \sigma}^{O E P}(r)$, reducing Eq. (37) to a symmetric eigenvalue problem. For example, in the Legendre pseudospectral method, the collocation points $\left\{x_{i}\right\}$ are the roots of the polynomials $P_{N+1}^{\prime}(x)$, where $N$ is the total number of grid points used in the discretization.

After the time-dependent single-electron wave functions $\left\{\psi_{i \sigma}\right\}$ are obtained, the total electron density $\rho(t)$ can be determined. The induced dipole moment can now be expressed as

$$
d(t)=\int \rho(\mathbf{r}, t) z d^{3} \mathbf{r}=\sum_{i \sigma}\left\langle\psi_{i \sigma}(\mathbf{r}, t)|z| \psi_{i \sigma}(\mathbf{r}, t)\right\rangle,
$$

$$
\begin{aligned}
d_{A}(t)= & \int \rho(\mathbf{r}, t) \frac{d^{2} z}{d t^{2}} d^{3} \mathbf{r} \\
= & \sum_{i, \sigma}\left\langle\psi_{i \sigma}(\mathbf{r}, t)\left|-\frac{\partial V_{S I C, \sigma}^{O E P}(\mathbf{r}, t)}{\partial z}+F g(t)\right|\right. \\
& \left.\times \psi_{i \sigma}(\mathbf{r}, t)\right\rangle
\end{aligned}
$$

in length form and acceleration form, respectively, where the second equality in Eq. (38) or (39) is valid within the single determinant approximation. The corresponding power spectrum can now be obtained by the Fourier transformation of the respective time-dependent dipole moment

$$
P(\omega)=\left|\frac{1}{t_{f}-t_{i}} \int_{t_{i}}^{t_{f}} d(t) e^{-i \omega t}\right|^{2}=|d(\omega)|^{2}
$$

and

$$
P_{A}(\omega)=\left|\frac{1}{t_{f}-t_{i}} \frac{1}{\omega^{2}} \int_{t_{i}}^{t_{f}} d_{A}(t) e^{-i \omega t}\right|^{2}=\left|d_{A}(\omega)\right|^{2}
$$

\section{EFFECT OF DYNAMICAL ELECTRON CORRELATION ON MULTIPLE HIGH-ORDER HARMONIC GENERATION OF He IN INTENSE LASER FIELDS: A CASE STUDY}

The study of multiple high-order harmonic generation in intense laser fields is a subject of much current interest both experimentally [35] and theoretically [3]. For the theoretical studies of HHG processes, the most successful timedependent approach so far is the single-active-electron approximation with a frozen core $[2,3]$. In this approach, however, electron correlation and core excitation, etc., cannot be explicitly treated. We note that in an earlier work, Kulander [27] has used the time-dependent Hartree-Fock method to perform a two-electron calculation of He multiphoton dynamics. However, only the multiphoton ionization process has been studied in [27]. In this work, the finite-difference algorithm with equal grid spacing is used to discretize the cylindrical coordinates. In a recent paper, Ullrich and Gross [28] have applied their time-dependent (TD) OEP-KLI method [9] to the study of the HHG of He, using the same numerical procedure (even grid spacing) described in [27]. However, no comparison of the TDOEP-KLI results with those of the SAE model is made in this latter work [28]. In this section, we shall extend the time-dependent OEP-KLISIC formalism and the generalized pseudospectral timepropagation method for a nonperturbative study of HHG of $\mathrm{He}$ atoms in intense laser fields. The results of both the SAE approximation and the all-electron calculations will be presented and compared to examine the range of validity of the SAE model. In the present study, the SAE calculation is performed within the time-dependent KLI-SIC formalism with the $1 s$ electron being held frozen. A comparison with relevant experimental data will be made. As will be shown below, the all-electron time-dependent KLI-SIC formalism allows a detailed exploration of the the effects of dynamical electron correlation or dynamical screening on both the ionization and HHG processes in a unified and self-consistent manner. 


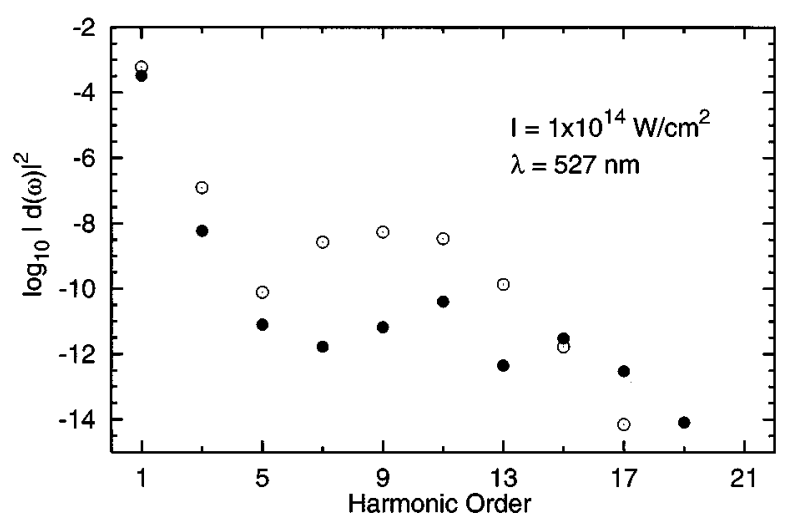

FIG. 1. Comparison of the HHG spectra of He atoms calculated by ALSDA (open circles) and TD-KLI-SIC-LSDA (filled circles) methods. The laser intensity used is $1 \times 10^{14} \mathrm{~W} / \mathrm{cm}^{2}$ and the wavelength used is $527 \mathrm{~nm}$.

For the special case of He, it can be shown that the TDKLI-SIC equation (14) can be formally reduced, in the exchange-only limit, to the time-dependent Hartree-Fock (TDHF) equation, since there are only $1 s$ spin orbitals being occupied initially. However, the present TD-KLI-SIC formalism is intended for general many-electron atoms. It is desirable to develop a general TD-KLI-SIC code for manyelectron atoms, including He. We have thus performed both TD-KLI-SIC and TDHF calculations for He. The results are essentially identical even though the detailed procedures involved are completely different. Such a comparison is useful and necessary, as it provides a benchmark for calibration of the accuracy of the general TD-KLI-SIC code for more complex atoms.

It is instructive to first make a comparison of the present TD-KLI-SIC procedure with the corresponding timedependent LSDA (or ALSDA) method. Due to the lack of long-range potential, the LSDA electron is too loosely bound with an ionization potential of 0.517 a.u. This is significantly lower than than the experimental value of 0.904 a.u. and the SIC-LSDA value of 0.918 a.u. For the case of laser wavelength $527 \mathrm{~nm}$, while the actual He atom requires the absorption of 11 photons from the ground state to reach the continuum, the LSDA electron only requires 6 photons to be ionized. Thus it is of interest to see the effect of the change of the ionization potential on the HHG spectrum. Figure 1 shows the comparison of the HHG power spectra of He atoms obtained from the TD-KLI-SIC-LSDA (filled circles) and ALSDA (open circles) calculations for the case of laser intensity $1 \times 10^{14} \mathrm{~W} / \mathrm{cm}^{2}$ and wavelength $527 \mathrm{~nm}$. The field is turned on using a $\sin ^{2}$ ramp for the first 10 optical cycles and the field amplitude is held constant afterward. The wave functions are propagated for 25 optical cycles and the HHG spectra are obtained from the Fourier transformation of the induced dipole moment (in length form) for the last 5 optical cycles. There are apparently significant differences between the two results both qualitatively and quantitatively: (i) The HHG intensities in the plateau regime of the ALSDA calculation are about 2-3 orders of magnitude larger than those of TD-KLI-SIC LSDA and (ii) the HHG cutoff position from the ALSDA occurs earlier than that of the TD-KLI-SIC LSDA. Such differences may be attributed to the typical behavior of looser bound LSDA electrons versus the tighter

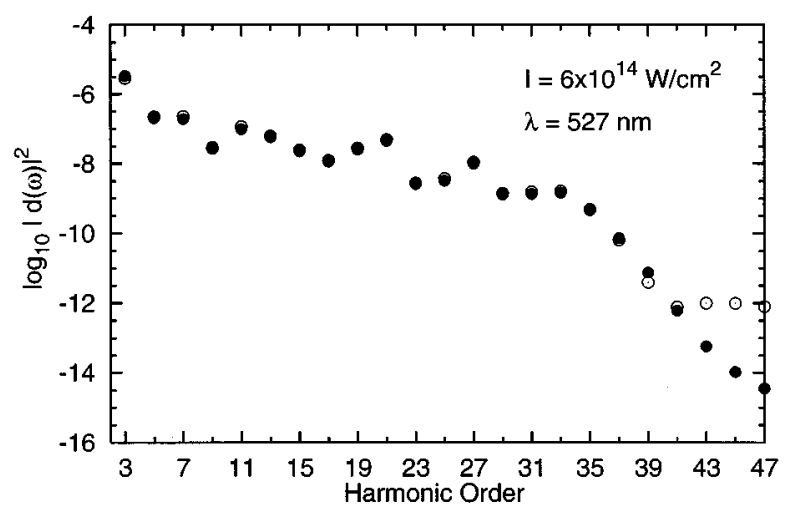

FIG. 2. Comparison of the HHG spectra of He atoms in length (open circles) and acceleration (filled circles) forms obtained from the TD-KLI-SIC method. The laser intensity used is $6 \times 10^{14} \mathrm{~W} / \mathrm{cm}^{2}$ and the wavelength used is $527 \mathrm{~nm}$.

bound SIC-LSDA electrons, namely, an atom with smaller ionization potential in general tends to produce a higher HHG plateau and earlier cutoff. For quantitative prediction of the HHG spectrum, it is thus essential to have the structure of the atoms (in this case, the long-range interaction and the ionization potential) correctly represented before pursuing the time-dependent calculations. Because of the relative simplicity and reasonably high accuracy, we have adopted the LSDA energy functional in our TD-KLI-SIC calculations discussed below.

Figure 2 shows the comparison of the HHG power spectra in length and acceleration forms for the case of a linearly polarized field with laser wavelength $527 \mathrm{~nm}$ and peak intensity $6 \times 10^{14} \mathrm{~W} / \mathrm{cm}^{2}$. The procedure for the field turning on is the same as that for Fig. 1. The total number of Legendre pseudospectral grid points used is 300 for the radial coordinate $r$ and a filter function [24] is used at some large distance (typically 50-70 a.u.) to filter out the ionizing wave packet. Up to 30 partial waves are used, which are sufficient to achieve the convergent results for the present case. Figure 2 shows that the HHG power spectrum in the length form nearly coincides (within a few percent) with that of the acceleration-form calculation throughout the plateau regime. Deviation occurs only well beyond the cutoff point where the acceleration-form results are believed to be more accurate $[26,36]$. The agreement of the length- and accelerationpower spectra in the physically important regime demonstrates that reasonably high-quality electron orbital wave functions can be achieved by the present TD-KLI-SIC formalism and the time-propagation numerical algorithm. In the following discussions, only the acceleration-form HHG power spectra will be presented. It is known that the acceleration form provides the more accurate framework for the description of the HHG processes when ionization becomes important [26,36].

In Figs. 3(a)-3(d), we show the comparison of the results of the all-electron time-dependent KLI-SIC (filled circles) and SAE-KLI-SIC (open circles) calculations for the case of laser wavelength $527 \mathrm{~nm}$ and laser intensity $I=10^{14}, 3 \times 10^{14}, 6 \times 10^{14}$, and $10^{15} \mathrm{~W} / \mathrm{cm}^{2}$, respectively. Several interesting features are noted. First, for the lowerintensity cases [Figs. 3(a) and 3(b)], there is good agreement of the SAE and all-electron HHG power spectra. The dis- 

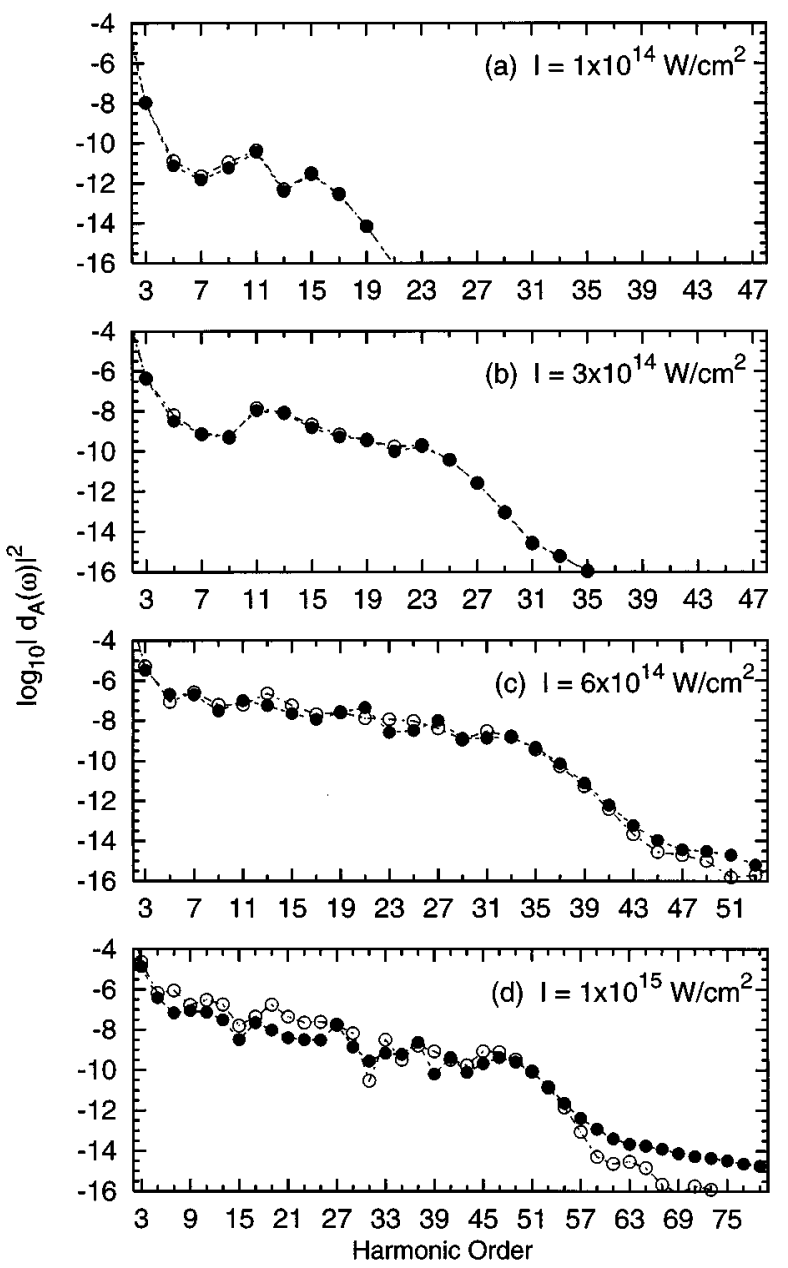

FIG. 3. Comparison of the HHG spectra of He atoms obtained from the all-electron calculations (filled circles) with those from the SAE model (open circles). (a)-(d) show the results corresponding to the laser intensity $I=10^{14}, 3 \times 10^{14}, 6 \times 10^{14}$, and $10^{15} \mathrm{~W} / \mathrm{cm}^{2}$, respectively. The laser wavelength used is $527 \mathrm{~nm}$.

crepancy increases with increasing laser intensity. For the highest-intensity case considered here [Fig. 3(d)], where ionization becomes appreciable, there is significant (1-2 orders of magnitude) departure of the SAE results from those of the all-electron calculations.

The most notable distinction of the SAE versus the twoelectron results in Fig. 3(d) arises in both the lower- and higher-harmonic regimes. The SAE model overestimates the harmonic-generation rates in the lower-energy harmonic regime, while significantly underestimates the higher-energy harmonics. To understand the origin of such a discrepancy, we show in Figs. 4(a)-4(c) the time-dependent bound-state population $N_{1 s}(t)$,

$$
N_{1 s}(t)=\int \phi_{1 s}^{*}(\mathbf{r}, t) \phi_{1 s}(\mathbf{r}, t) d \mathbf{r}=\frac{N(t)}{2},
$$

of the He atom for the case of $527 \mathrm{~nm}$ and three different laser peak intensities: (a) $3 \times 10^{14} \mathrm{~W} / \mathrm{cm}^{2}$, (b) $6 \times 10^{14} \mathrm{~W} / \mathrm{cm}^{2}$, and (c) $1 \times 10^{15} \mathrm{~W} / \mathrm{cm}^{2}$, respectively. (In both Figs. 3 and 4 , we use a $\sin ^{2}$ ramp for the first ten optical cycles and the field amplitude is held constant afterward.) Note that the two $1 s$ electrons (spin up and down) in the $\mathrm{He}$
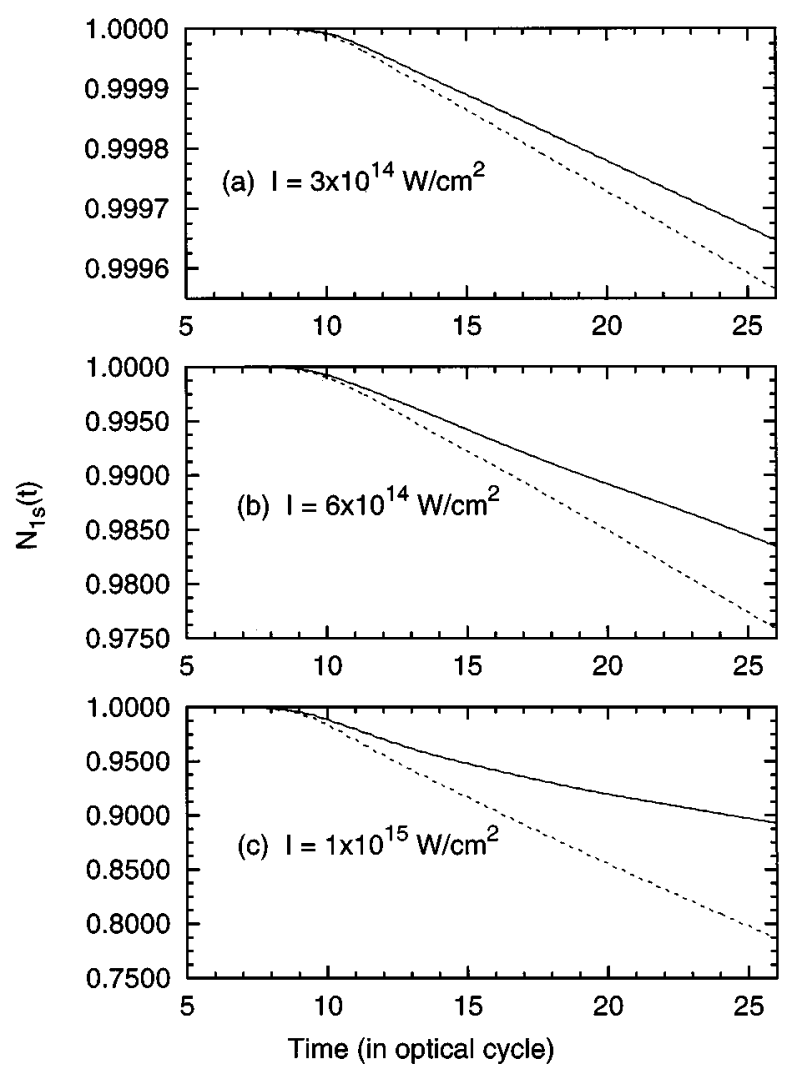

FIG. 4. Comparison of the bound-state populations $N_{1 s}(t)$ of $\mathrm{He}$ atoms obtained from the all-electron calculations (solid lines) with those from the SAE model (dashed lines) for several laser intensities. The laser wavelength used is $527 \mathrm{~nm}$.

atom are completely equivalent and the spin index can be ignored in Eq. (42). The physical meaning of $N_{1 s}(t)$ is the total electron probability (density) within the finite regime bounded by the filter. For the case of two-electron timedependent KLI-SIC calculations, $N_{1 s}(t)$ includes the total bound electron probability due to the neutral $\mathrm{He}$ atom and the $\mathrm{He}^{+}$ion all together. In the SAE model, $N_{1 s}(t)$ represents only the probability of the "active" electron bounded to the neutral He atom, while the other $1 s$ electron is frozen to the $1 \mathrm{~s}$ orbital. The ionization rates can be estimated from the slopes of the $N_{1 s}(t)$ vs time plots in Figs. 4(a)-4(c). It is seen that the SAE model always predicts larger ionization rates than those of the two-electron calculations and the discrepancy increases with increasing laser intensity. We note that in the actual time-dependent ionization process of the He atom, the average electron binding energy of the atom should increase with time. This is because when the atom is partially ionized (in terms of the ionization probability), the remaining electrons (in terms of bound electron probability) will be more tightly bounded by the nucleus due to the dynamical screening effect. For example, the ionization potential of the neutral $\mathrm{He}$ atom is 0.904 a.u., while for the $\mathrm{He}^{+}$ion the binding energy is 2.0 a.u. The all-electron TDDFT takes into account such a dynamical screening effect or equivalently the dynamical electron correlation, while the SAE model does not. In the SAE model, the ionization potential is a static quantity since the remaining electron is frozen. As a result, the SAE electron is always less bounded by the nucleus, leading to the overestimation of the ionization rate. 
A similar argument can be applied to the explanation of the discrepancy of the all-electron and SAE HHG results in Figs. $3(c)$ and 3(d). In general, for a given laser intensity and frequency, an electron with smaller (larger) ionization potential tends to produce larger (smaller) harmonic-generation rates for lower harmonics but with a shorter (longer) cutoff in the higher-harmonics regime. Since the active electron in the SAE model has smaller ionization potential than that in the all-electron theory when ionization occurs, the SAE model tends to predict earlier HHG cutoff, as can be seen in Figs. $3(\mathrm{c})$ and 3(d).

In this context, it is instructive to pursue further exploration of the effect of dynamical electron correlation on the production of higher harmonics. This is prompted by a recent experimental study of the HHG of the He atom by Sarukura et al. [37], using an ultrahigh-power $\mathrm{KrF}$ laser $(248.6 \mathrm{~nm})$ with a peak intensity of $2 \times 10^{17} \mathrm{~W} / \mathrm{cm}^{2}$. Due to the possible effect of defocusing of the laser beam, the actual effective peak intensity responsible for the HHG production is estimated to be about $3.5 \times 10^{15} \mathrm{~W} / \mathrm{cm}^{2}$ [38]. To fit the experimental data (from 9th to 23rd harmonics), several theoretical works found that it was necessary to use a two-step model $[38,39]$. The fitting of the lower harmonics (up to the 13th-15th harmonic) was achieved by the SAE model for the neutral $\mathrm{He}$ atom, while a separate $\mathrm{He}^{+}$model was used to fit the higher harmonics. In essence, in the two-step model the HHG of $\mathrm{He}$ is considered to be purely two separate oneelectron phenomena. In a recent paper [40] using the twoelectron wave-function approach, a different interpretation of the He HHG data was inferred: The entire HHG spectrum of $\mathrm{He}$ is due to the neutral $\mathrm{He}$ atom alone and the higher harmonics are the result of strong electron correlations. However, in this latter work, only the wave functions of the ${ }^{1} S$ and ${ }^{1} P$ states are included and no ionization information is provided. Thus it appears that the actual mechanism responsible for the observed higher harmonics in $\mathrm{He}$ [37] is still not yet settled.

To explore the origin of the observed HHG behavior [37] and to resolve the discrepancy of the two different theoretical interpretations [38-40] of the experimental results, we present below a detailed analysis of both the ionization and HHG processes using the time-dependent KLI-SIC formalism. Following Ref. [38], we consider a laser field (248.6 $\mathrm{nm})$ with a $\sin ^{2}$ pulse of 128 optical cycles (105 fs) and a peak intensity of $3.5 \times 10^{15} \mathrm{~W} / \mathrm{cm}^{2}$. Figure $5(\mathrm{a})$ shows the time-dependent population $N_{1 s}(t)$ and the laser pulse profile. It is seen that in the SAE model (thin line), the neutral He atom is completely ionized to the $\mathrm{He}^{+}$ion before the peak intensity is reached. The two-electron TDDFT calculation (thick line), however, shows quite different behavior. We discuss below the implications of the behavior of the $N_{1 s}(t)$ curve (two-electron calculations) in Fig. 5(a). First note that $N_{1 s}(t)=1.0$ means that all the electrons are bounded to the neutral He atom and that there are no ions. When $N_{1 s}(t)=0$, all the electrons are ionized, and this corresponds to the $\mathrm{He}$ ${ }^{2+}$ ion limit. For the intermediate case, $N_{1 s}(t)=0.5$, it implies that on average one electron has been ionized and $\mathrm{He}^{+}$ is the dominant species, though there could still have been some neutral $\mathrm{He}$ atom and $\mathrm{He}^{2+}$ ion exist. Figure 5(a) shows that $N_{1 s}(t)$ stays close to 1.0 during the rising part of the pulse (from $t=0$ to about 30 optical cycles) and
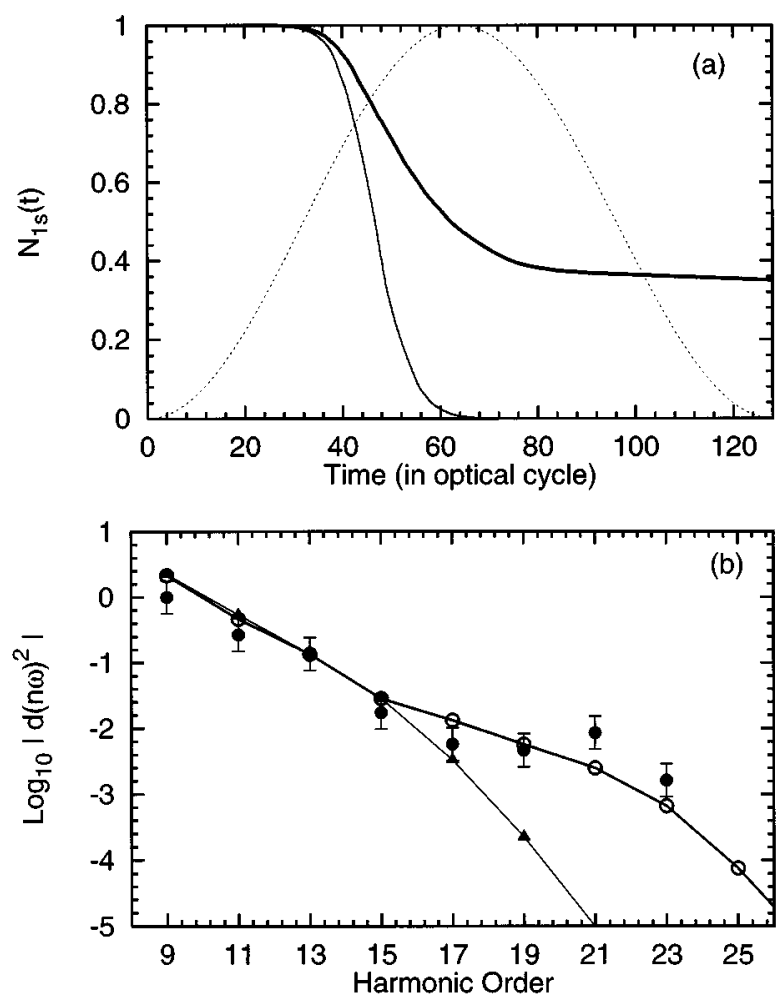

FIG. 5. (a) Comparison of the bound-state populations $N_{1 s}(t)$ of $\mathrm{He}$ atoms obtained from the all-electron calculations (thick lines) with those from the SAE model (thin lines). The envelope of the laser pulse is also presented (dashed line). (b) The HHG spectrum of He obtained from the all-electron calculation (open circles) and from the SAE model (filled triangles). The experimental data (with error bars) are also shown for comparison. The HHG yields are normalized to the 13th harmonic peak. In both (a) and (b), the laser peak intensity used in the calculation is $I=3.5 \times 10^{15} \mathrm{~W} / \mathrm{cm}^{2}$ and the wavelength $\lambda=248.6 \mathrm{~nm}$.

there is little ionization produced. As the field increases from 30 to about 60 optical cycles and approaches the peak intensity, $N_{1 s}(t)$ drops rapidly from 1.0 to about 0.5 . During this period, the neutral He atom is rapidly ionized to $\mathrm{He}^{+}$and the $\mathrm{He}^{+}$ion becomes the dominant species. After the peak intensity is over (60-80 optical cycles), the decay of $N_{1 s}(t)$ continues, but with a slower rate due to the decrease of the laser intensity. After about 80 optical cycles, $N_{1 s}(t)$ drops much more slowly and nearly approaches a constant. This can be attributed to the fact as the laser intensity is further decreased, $\mathrm{He}^{+}$, the dominant species, becomes much more difficult to ionize due to its substantially larger ionization potential than that of the neutral $\mathrm{He}$ atom.

Figure 5(b) shows the corresponding harmonic-generation power spectra obtained, respectively, by the two-electron calculations (open circles) and the SAE model (solid triangles). Also shown are the experimental data [37] (normalized to the 13th harmonic) (solid circles with error bars) for comparison. Similar to the previous theoretical studies [3840], we found that the SAE HHG spectrum shows a large departure (underestimation) from the experimental data for harmonics higher than the 15th harmonic. However, our twoelectron TDDFT results agree well with the whole experimental HHG spectrum. From the analysis of the data in Fig. 5 (a), we can draw the following conclusion on the actual 
mechanism responsible for the the observed higher harmonics in $\mathrm{He}$ [37]: Both the $\mathrm{He}^{+}$ion and the dynamical electron correlation are important to the production of the higher harmonics. In fact, for the present case, these two factors are related to each other to some extent. The production of the higher harmonics is clearly a two-electron correlated phenomenon because the SAE model fails completely in this high-intensity regime, whereas the two-electron TDDFT provides the correct result. However, the production of the higher harmonics appears not entirely due to the neutral $\mathrm{He}$ atom as inferred in a recent work [40]. In fact, Fig. 5(a) shows that while both the neutral $\mathrm{He}$ atom and the $\mathrm{He}^{+}$ion contribute to the HHG processes, the $\mathrm{He}^{+}$ion is the dominant species just before and after the peak intensity and is the one likely to be making the major contributions to the production of the higher harmonics. The present TDDFT thus provides a unified and self-consistent dynamical picture of the origin of the generation of the higher harmonics in He.

We are currently extending the time-dependent KLI-SIC formalism to the study of multiphoton dynamics of more complex atoms in intense laser fields. Results will be presented elsewhere.

\section{ACKNOWLEDGMENTS}

The calculations in this work were performed using the Origin2000 supercomputer sponsored by the Kansas Center for Advanced Scientific Computing. We thank Dr. E. K. U. Gross for his constructive comments on an earlier version of this paper.
[1] See, for example, J. Parker, K. T. Taylor, C. W. Clark, and S. Blodgett-Ford, J. Phys. B 29, L33 (1996); J. Zhang and P. Lambropoulos, Phys. Rev. Lett. 77, 2186 (1996); J. Purvis et al., ibid. 71, 3943 (1993); C. Laughlin and S. I. Chu (unpublished).

[2] J. L. Krause, K. J. Schafer, and K. C. Kulander, Phys. Rev. A 45, 4998 (1992); Phys. Rev. Lett. 68, 3535 (1992).

[3] For a review see A. L'Huillier, K. J. Schafer, and K. C. Kulander, J. Phys. B. 24, 3315 (1991).

[4] P. Hohenberg and W. Kohn, Phys. Rev. 136, B864 (1964).

[5] W. Kohn and L. J. Sham, Phys. Rev. 140, A1133 (1965).

[6] E. Runge and E. K. U. Gross, Phys. Rev. Lett. 52, 997 (1984).

[7] G. D. Mahan and K. R. Subbaswamy, Local Density Theory of Polarizability (Plenum, New York, 1990).

[8] E. K. U. Gross and W. Kohn, Adv. Quantum Chem. 21, 255 (1990).

[9] C. A. Ullrich, U. J. Gossmann, and E. K. U. Gross, Phys. Rev. Lett. 74, 872 (1995).

[10] D. Telnov and S. I. Chu, Chem. Phys. Lett. 264, 466 (1997).

[11] For reviews on generalized Floquet formalisms, see S. I. Chu, Adv. At. Mol. Phys. 21, 197 (1985); Adv. Chem. Phys. 73, 739 (1989).

[12] See, for example, R. Parr and W. Yang, Density-Function Theory of Atoms and Molecules (Oxford University Press, New York, 1989).

[13] S. J. Vosko, L. Wilk, and M. Nusair, Can. J. Phys. 58, 1200 (1980).

[14] A. D. Becke, Phys. Rev. A 38, 3098 (1988).

[15] C. Lee, W. Yang, and R. G. Parr, Phys. Rev. B 37, 785 (1988).

[16] J. P. Perdew and Y. Wang, Phys. Rev. B 33, 8800 (1986).

[17] T. Grabo and E. Gross, Chem. Phys. Lett. 240, 141 (1995).

[18] X. M. Tong and S. I. Chu, Phys. Rev. A 55, 3406 (1997).

[19] J. B. Krieger, Y. Li, and G. J. Iafrate, Phys. Lett. A 146, 256 (1990); J. Krieger, Y. Li, and G. Iafrate, Phys. Rev. A 46, 5453 (1992); Y. Li, J. B. Krieger, and G. J. Iafrate, ibid. 47, 165 (1993).
[20] R. T. Sharp and G. K. Horton, Phys. Rev. 90, 317 (1953).

[21] J. D. Talman and W. F. Shadwick, Phys. Rev. A 14, 36 (1976).

[22] J. P. Perdew and A. Zunger, Phys. Rev. B 23, 5048 (1981).

[23] J. Chen, J. Krieger, Y. Li, and G. Iafrate, Phys. Rev. A 54, 3939 (1996).

[24] X. M. Tong and S. I. Chu, Chem. Phys. 217, 119 (1997).

[25] M. R. Hermann and J. A. Fleck, Jr., Phys. Rev. A 38, 6000 (1988).

[26] T. F. Jiang and S. I. Chu, Phys. Rev. A 46, 7322 (1992).

[27] K. C. Kulander, Phys. Rev. A 36, 2726 (1987).

[28] C. A. Ullrich and E. K. U. Gross, Comments At. Mol. Phys. 33, 211 (1997).

[29] J. Wang, S. I. Chu, and C. Laughlin, Phys. Rev. A 50, 3208 (1994).

[30] G. Yao and S. I. Chu, Chem. Phys. Lett. 204, 381 (1993).

[31] D. Telnov and S. I. Chu, J. Phys. B 29, 4401 (1996).

[32] See, for example, G. Yao and S. I. Chu, Chem. Phys. Lett. 197, 413 (1992); J. Wang and S. I. Chu, ibid. 227, 663 (1994); D. Telnov and S. I. Chu, ibid. 255, 223 (1996).

[33] M. D. Feit, J. A. Fleck, Jr., and A. Steiger, J. Comput. Phys. 47, 412 (1982).

[34] C. Canuto, M. Y. Hussaini, A. Quarteroni, and T. A. Zang, Spectral Methods in Fluid Dynamics (Springer, Berlin, 1988).

[35] For a review see A. L'Huillier, L. A. Lompre, G. Mainfray, and C. Manus, Adv. At. Mol. Opt. Phys. Suppl. 1, 139 (1992).

[36] K. Burnett, V. C. Reed, J. Cooper, and P. L. Knight, Phys. Rev. A 45, 3347 (1992).

[37] N. Sarukura et al., Phys. Rev. A 43, 1669 (1991).

[38] A. Sanpera, P. Jonsson, J. B. Watson, and K. Burnett, Phys. Rev. A 51, 3148 (1995).

[39] H. Xu, X. Tang, and P. Lambropoulos, Phys. Rev. A 46, R2225 (1992).

[40] N. Moiseyev and F. Weinhold, Phys. Rev. Lett. 78, 2100 (1997). 